
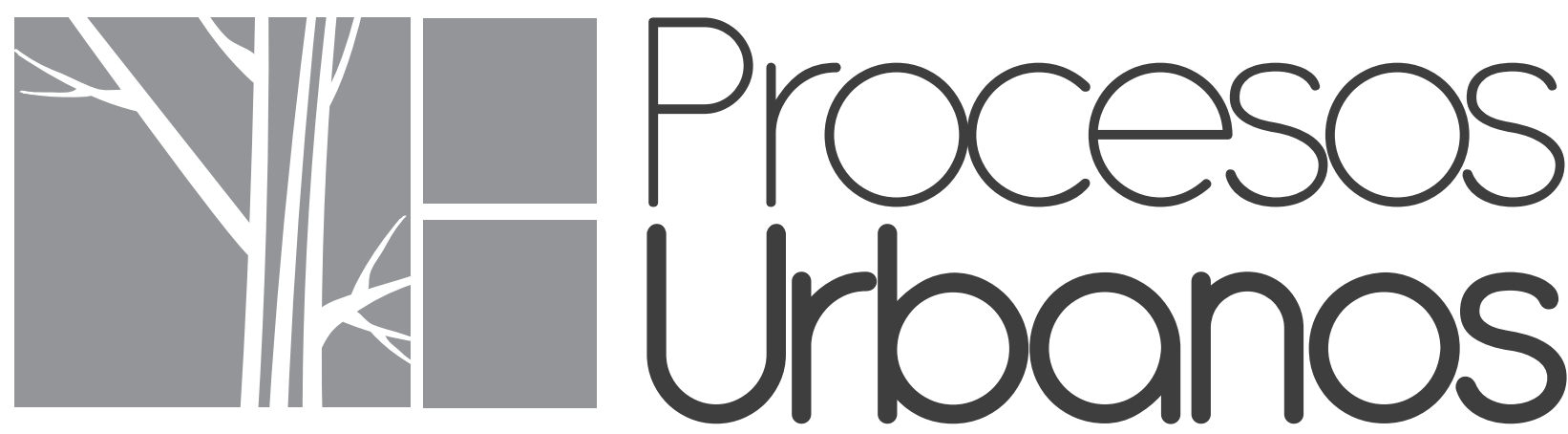

Revista de Divulgación Científica 


\section{Revista Procesos Urbanos}

Facultad de Ciencias Básicas, Ingeniería y Arquitectura.

ISSN: 2422-085X

Sincelejo, Enero - Diciembre de 2014

Piedad Martínez Carazo

Rectora

\section{Lidia Flórez de Albis}

Vicerrectora Académica

\section{Jhon Víctor Vidal}

Director de Investigaciones

\section{Pedro Arturo Martínez Osorio}

Editor

\section{Comité Editorial:}

PhD. Alexander Niño Soto. Universidad Antonio Nariño

PhD. Jorge Ramírez Nieto. Universidad Nacional de Colombia

Msc. Juan Carlos Pergolis. Universidad Católica de Colombia

PhD. Piedad Martínez Carazo. Corporación Universitaria del Caribe, CECAR

PhD. Emiro F. Martínez. Universidad de York, Canadá

\section{Comité Científico:}

Msc. Gloria Aponte García. UPB, Medellín

Msc. Jorge Vásquez Muñoz. UPB, Medellín

PhD. Jorge Gómez Ricardo, Universidad del Magdalena

\section{Editorial CECAR}

\section{Libia Narváez Barbosa}

Coordinadora Editorial

\section{Eduardo Támara Galván}

Corrector de Estilo

\section{Alejandrina Jaramillo y Roger Goez Castillo}

Diseño Gráfico

Diagramación e Impresión:GRÁFICAS DEL CARIBE S.A.S.

Cra. 1B No. 40-42 Montería Tel. (57) (4) 7826622 Telefax (57) (4) 7817112

Email: diseno@graficaribe.co

\section{Dirección:}

Carretera Troncal de Occidente

Kilómetro 1 Vía a Corozal

Tel: 280402928040172804018 Ext 1115

www.cecar.edu.co

http://cecar.edu.co/revista-procesos-urbanos 


\title{
LA IMPORTANCIA DEL CONCEPTO Y SU ARGUMENTACIÓN EN EL DISEÑO ARQUITECTÓNICO
}

\author{
The importance of the concept and argument in architectural design
}

Carlos G. Buelvas Meza ${ }^{1}$

Fecha: Recibido Marzo 10 de 2014 / Aceptado Junio 24 de 2014

\section{RESUMEN}

El Concepto es el hilo conductor que guía al proyecto arquitectónico en todas sus etapas de desarrollo. El objetivo de este trabajo es presentar una reflexión sobre la importancia que tiene el concepto en el proceso de diseñoy cómo afrontar su argumentación. El método con el cual se planteó la investigación, parte de un análisis a partir de la revisión de fuentes bibliográficas, que permitieran la identificación de los elementos de juicio que soportaran el fundamento teórico, de la importancia que tiene el concepto arquitectónico y su posterior argumentación, como etapas previas al desarrollo del proyecto. Entre los resultados se destaca el entendimiento de la argumentación como el mecanismo que permite el proceso reflexivo de la conceptualización en el proceso de diseño, a través de la complementación con las experiencias, permite el desarrollo de las ideas. Su producción es además un proceso de interacción con el interlocutor, indispensable para que exista comunicación desde el punto de vista intelectual. Como conclusión más importante se presenta, la importancia de la argumentación en el proceso de diseño, ésta surge de la necesidad de comunicación y defensa de las ideas a través del mecanismo de relacionar datos concretos con las abstracciones y generalizaciones. El diseño arquitectónico es el resultado del concepto y no el proceso inverso.

Palabras Clave: Concepto, idea, conceptualización, argumentación, proyecto arquitectónico.

\begin{abstract}
The Concept is the conductive thread that he guides the architectural project in all his stages of development. The aim of this work is to present a reflection on the importance that has the concept in the process of design and how to confront his argumentation. The method in the research, part of an analysis from the review of bibliographical sources, which were allowing the identification of the facts that were supporting the theoretical foundation, of the importance that has the architectural concept and his later argumentation, as stages before the development of the Project. Between the results we can see that the understanding of the argumentation is outlined as the mechanism that allows the reflexive process
\end{abstract}

\footnotetext{
${ }^{1}$ Arquitecto CECAR. Magister en administración Universidad Nacional. Corporación Universitaria del Caribe, Facultad de Ciencias Económicas, Grupo de investigación: Estudios Socioeconómicos, Administrativos y Contables, Sincelejo - Sucre. Email: carlos.buelvas@cecar.edu.co
} 
PROCESOS URBANOS - Revista de Divulgación Científica Vol. 1 Enero - Diciembre 2014 (35 - 46)

of the conceptualization in the process of design, across to join with the experiences, allows the development of the ideas. His production is in addition a process of interaction with the speaker, indispensably in order that communication exists from the intellectual point of view. The more important conclusions it is presents the importance of the argumentation in the process of design, this one arises from the need of communication and defense of the ideas across the mechanism of relating concrete information to the abstractions and generalizations. The architectural design is the result of the concept and not the inverse process.

Keywords: Concept, ideas, conceptualization, argumentation, architectural project.

\section{INTRODUCCIÓN}

La globalización con su vertiginosa oleada de innovaciones y cambios sobrepasa toda capacidad de adaptación de los seres humanos. Para contrarrestar sus efectos, cada vez más se imponen obligaciones que menoscaban la calidad de vida; por ello al pensar en el concepto arquitectónico y la estrategia de argumentación, se va quedando rezagado hasta llegar a la moda en que primero se diseña y después se le imprime el concepto al proyecto.

Se ha olvidado que el concepto es el eje transversal que guiará al proyecto en todas las etapas de su desarrollo, es el hilo conductor y la esencia, sin embargo, se quiere imprimir el espíritu al diseño, siendo que el proceso es al revés; es el espíritu el que le da vida al diseño.

Este artículo pretende presentar una reflexión que llame la atención sobre la importancia que tiene el concepto en el diseño, y del desarrollo del concepto hasta llegar a la argumentación propia, establecer que, tanto el concepto como la argumentación, tienen unos elementos reconocibles que le dan peso al proceso de diseño. Por último, se presentan algunos conceptos que permiten la materialización de la argumentación teórica.

\section{METODOLOGÍA}

Este trabajo es producto de la investigación titulada, "Educación, concepto y cultura de paz en la construcción del discurso social", y a través de él, se construyen puntos de acercamiento con otras ciencias sociales haciendo énfasis en la importancia de la educación y el concepto, como elementos claves de las definiciones claras y precisas que se necesitan para iniciar procesos exitosos, a través de la argumentación. Esta se planteó como una reflexión desde el paradigma cuantitativo de tipo descriptivo complementada con una revisión bibliográfica de fuentes primarias y secundarias.

El primer paso fue la ubicación de los documentos bibliográficos consultando varias fuentes documentales, se realizó una revisión de la literatura contenida en las bases de datos Scielo y Google Académico, utilizando los descriptores: "concepto arquitectónico, argumentación de proyectos", También se utilizaron las fuentes primarias de la biblioteca Gerónimo Osiris, de la Corporación Universitaria del Caribe CECAR, de donde se obtuvo un total de 35 registros y 5 libros, de los cuales se clasificaron para su estudio 5 documentos de las bases de datos y 3 libros.

El artículo se planteó desde la óptica analítica crítica como una reflexión al tema de la importancia del concepto y su argumentación. Para la búsqueda de la bibliografía no se determinó ninguna restricción, por lo que se pudo hacer de forma automatizada y manual. Como parámetro de selección de los documentos 
se estableció la pertinencia con el tema, la identificación y correlación de hechos o sucesos que permitieran establecer una secuencia cronológica del tema investigado.

Identificados los documentos, se sometieron a un proceso de análisis, para extraer la información más relevante contenida en esa fuente de información; posteriormente se realizó una síntesis, proceso mediante el cual se procede a la condensación y destilación de la información analizada de una o más fuentes. Por último se procedió a la redacción del artículo.

\section{RESULTADOS Y DISCUSIÓN}

El concepto, es uno de los elementos de mayor peso en los diseños arquitectónicos, sin embargo, la forma como se aborda en los proyectos da pie para pensar que se está mecanizando o simplemente, no se tiene claridad de su significado y se toma de manera a priori. Por lo anterior, es necesario establecer las bases desde su significancia y construir un marco teórico que permita situar en el pedestal que se merece, este elemento de la arquitectura.

Las creaciones intelectuales del hombre necesitan un proceso reflexivo por medio del cual, se dé explicación objetiva, de la decisión que impulsa a la invención; es la justificación del acto creador, lo cual es imposible sin un concepto. Todo acto creador es sustentado por uno o varios conceptos, los cuales le imprimen el carácter de veracidad y utilidad. Siendo la arquitectura, por excelencia, una ciencia creadora, necesita retomar lo importante del concepto en sus diseños.

El diccionario de la Real Academia Española presenta las definiciones más simples de lo que es un concepto. (DRAE, 2013)

(Del lat. conceptus).

1. adj. ant. Conceptuoso.
2. m. Idea que concibe o forma el entendimiento.

3. m. Pensamiento expresado con palabras.

4. m. Sentencia, agudeza, dicho ingenioso.

5. m. Opinión, juicio.

Expresado de esta forma, el concepto implica un proceso mental de apropiación del conocimiento que posteriormente se necesita para explicar las decisiones que se toman.

De forma general, "Los conceptos son constructos que permiten definir objetos o fenómenos de la sociedad, la naturaleza y el pensamiento, los que coherentemente articulados dan lugar a las ideas como expresión de relaciones teóricas, prácticas, metodológicas o simbólicas". (Cruz, S. sf. Pág. 2). Este fragmento permite ver la materialización del concepto, en forma de definiciones, las cuales organizadas dan origen a las ideas.

Concretamente, en arquitectura se define como, ".... Es una idea que guía el proceso de diseño, y sirve para asegurar una o varias cualidades del proyecto: imagen, funcionalidad, economía, mensaje...". (Miranda, E. 2011). En concordancia con lo anterior, el concepto es valorado como una idea que tiene la utilidad de guiar todo el proceso de diseño, en esa misma línea de pensamiento es definido el concepto como "..esa parte hipotética de la fase proyectiva dentro del proceso de investigación donde la idea aparece en su estado original..." (Martínez, 2009).

Sin embargo, el materialismo dialéctico tiene otra forma de observar el concepto:

"El concepto no es el punto de partida del conocimiento, sino su resultado. (...) el materialismo dialéctico considera que el concepto es una forma peculiar de reflejo de los objetos, de las cosas del mundo material y de las leyes de su movimiento. Los conceptos son objetivos por su contenido. Incluso los conceptos más abstractos, tienen sus analogías, sus prototipos en el mundo objetivo. 


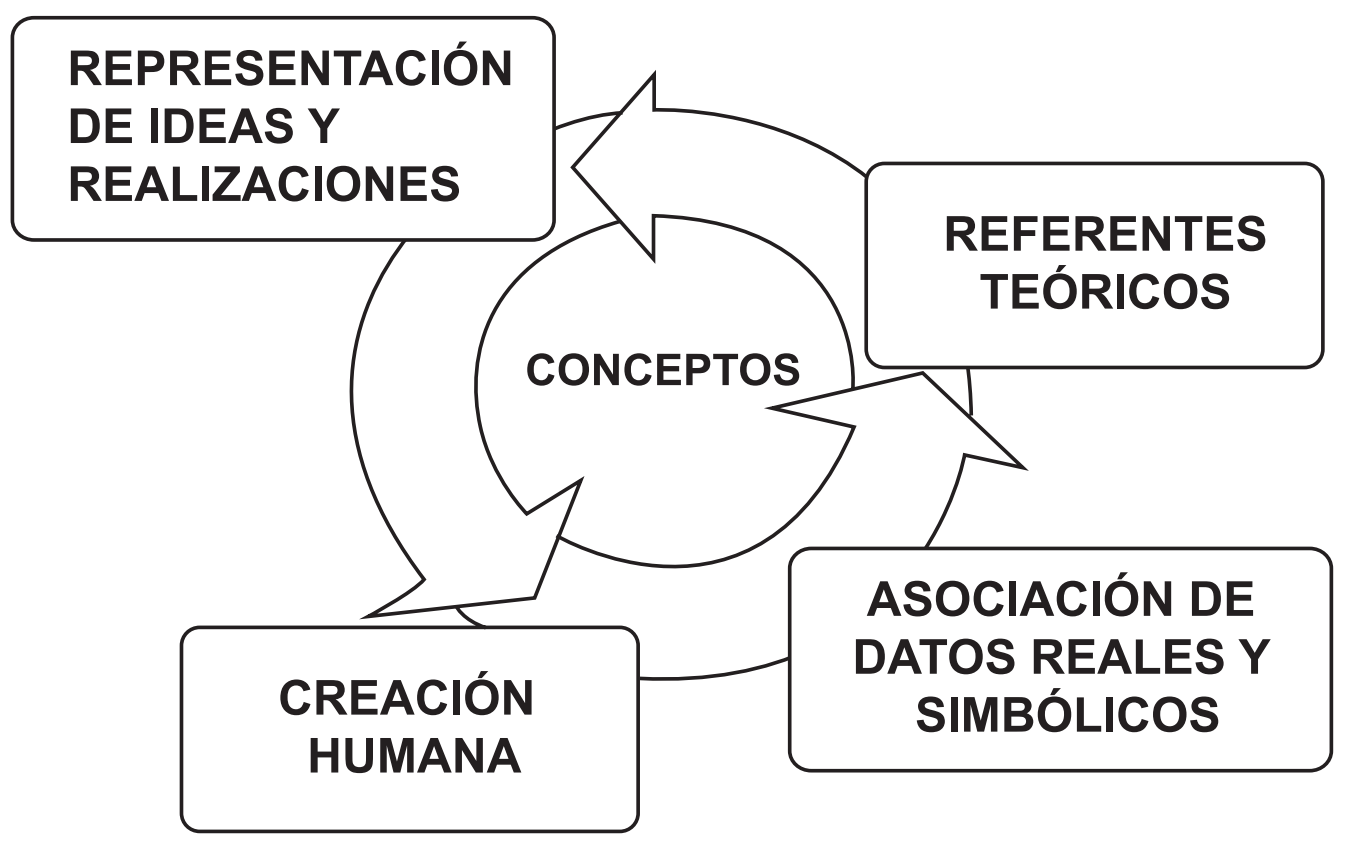

Figura 01 Relaciones que dan lugar a los conceptos. Fuente: Cruz, S. sf. Pág. 3

En el concepto se refleja el contenido encerrado en las cosas." (Kopnin, V. sf. Pág. 229).

Esta definición materializa mucho más al concepto, afirmando que aun los conceptos más abstractos tienen semejantes en el mundo real, esto se debe en parte, a la necesidad que existe en el campo intelectual, de explicar de forma sistemática las características de los objetos de estudio. Entonces, el punto de partida para tener la noción de concepto, es la experiencia desde la óptica de los saberes adquiridos y complementados con el análisis del objeto de estudio; de esta forma llegamos a la afirmación que los conceptos tienen una base empírica, porque se tiende a generalizar desde la particularidad de la experiencia. "sin la acumulación de un determinado material empírico es imposible formar un solo concepto." (Kopnin, V. sf).

Sin embargo, creer que el concepto solo encierra ese componente empírico, es transitar por el terreno de la simplicidad, porque se necesitan procesos mentales complejos para hacer abstracciones y relacionarlas entre sí, para explicar el mundo objetivo a través de generalizaciones, por lo tanto, también tiene un componente cualitativo. "De manera que conceptualizar es un proceso de construcción de ideas sobre la base de experiencias y vivencias, que a través de procesos lógicos, se van configurando en definiciones precisas de cualidades esenciales de los objetos y fenómenos de la realidad objetiva". (Cruz, S. sf. Pág. 3).

En la realidad objetiva se encuentran multiplicidad de problemas las cuales requieren ser conceptualizadas para su comprensión y búsqueda de soluciones,

Todo concepto remite a un problema, a unos problemas sin los cuales carecería de sentido, y que a su vez sólo pueden ser despejados o comprendidos a medida que se vayan solucionando... sólo se crean conceptos en función de los problemas que se consideran mal vistos o planteados. (Guattari, F. 1999. Pág 22). 
Lo anterior nos remite a la forma utilitaria del concepto, el cual lleva implícitamente la solución de problemas, sin ello carecería de sentido, por lo que el concepto cobra importancia en la medida de su utilidad; en este sentido y visto desde la arquitectura "Las necesidades analizadas en el planteamiento del problema, se convierten en lo que llamamos un esquema básico o anteproyecto de acuerdo con su nivel de definición y profundización. (Martínez, 2009). Esquema básico o anteproyecto entendidos como el inicio de la solución del problema.

En el desarrollo de la arquitectura se ha utilizado variedad de conceptos en su práctica, pero desde un punto de vista general, se puede clasificar en tres grandes campos, así: "Compositivos, sensibles y problemáticos, cada uno con implicaciones y sistemas de trabajo que enfatizan en aspectos diferentes; y como antes se ha dicho, puede que se integren o formen un conjunto de ideas complejas". (Martínez, 2009).

Los conceptos compositivos tienen referencia al orden y a la organización, "son todos aquellos que aluden al orden de las ideas abstractas de la geometría y la organización de las formas arquitectónicas, como por ejemplo, el eje, la simetría, la rotación," (Martínez, 2009). Sin embargo,

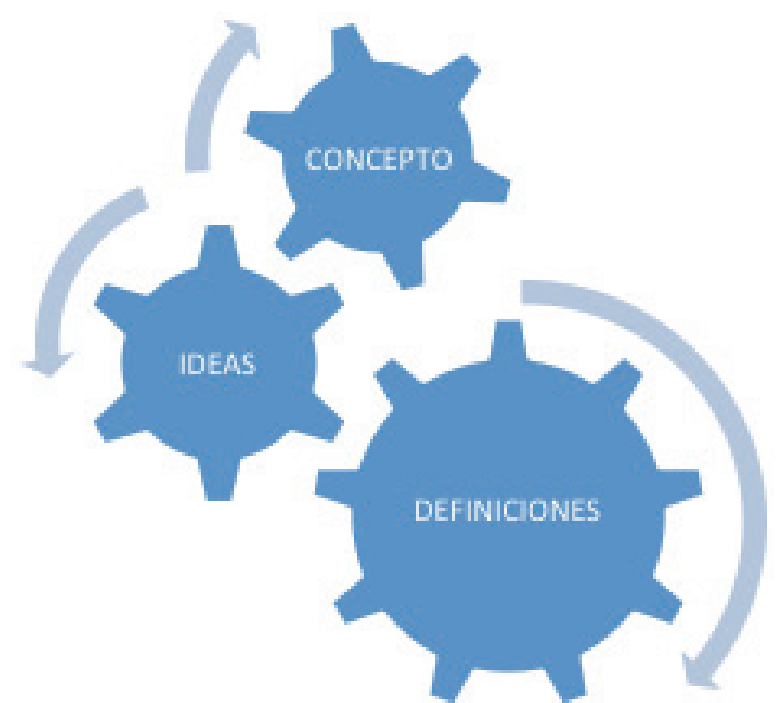

Figura 02 Proceso de Conceptualizar.

Fuente: Elaboración propia del autor

los principios anotados por el autor se complementan con los siguientes: jerarquía, ritmo, repetición, pauta, transformación, transición, unidad, directriz, equilibrio, sustracción.

Los conceptos sensibles plantean soluciones desde la perspectiva emocional del ser interior, dando relevancia a los sentimientos y la parte intangible "...buscan plantear soluciones a los problemas desde una perspectiva interiorista de los sentimientos y percepciones del arquitecto, entendiendo estas desde los puntos de vista sensitivo

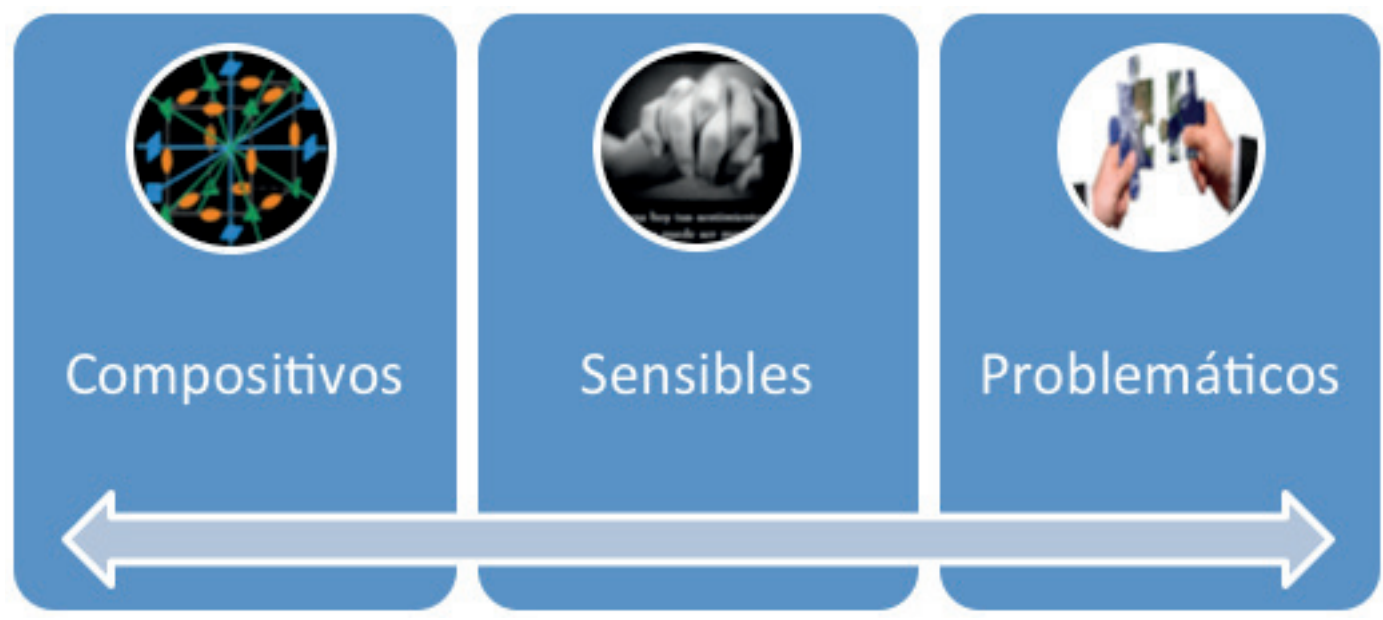

Figura 03 Clasificación del concepto.

Fuente: Elaboración propia del autor 


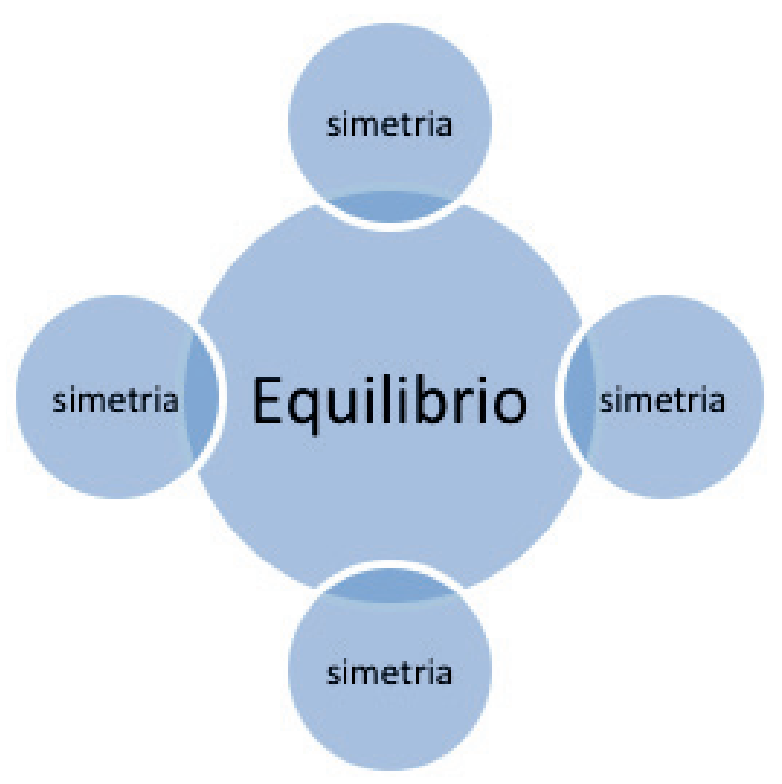

Figura 04 Esquema conceptos compositivos. Fuente: Elaboración propia del autor

y sensorial, es decir, lo que sientes emotivamente, y lo que sientes a través de los sentidos". (Martínez, 2009). Ejemplos de estos conceptos son las metáforas de la pureza, paz, eternidad, reposo, luz, oscuridad. etc.

Los conceptos problémicos son más particulares y específicos porque se desprenden de la información que se origina del mismo problema, "Son un tanto más complejos y provienen de los datos

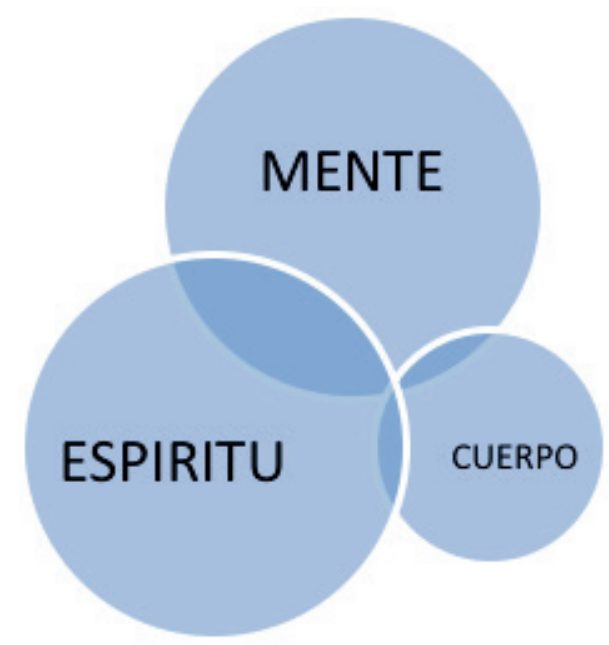

Figura 06 Esquema Conceptos sensibles. Fuente: Elaboración propia del autor

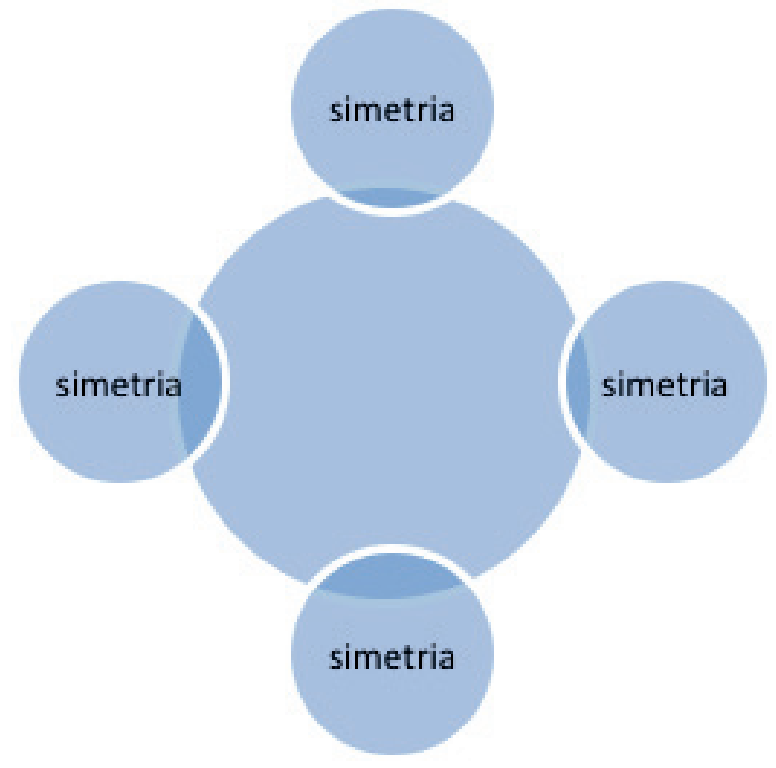

Figura 05 Esquema conceptos compositivos. Fuente: Elaboración propia del autor

mismos que arroja el problema; es decir, trabajan con las ideas directas que surgen de las necesidades mismas del problema". (Martínez, 2009).

Manejar un marco conceptual amplio, posibilita las condiciones para explicar de mejor forma los resultados para ser congruentes con la argumentación, debido a que esta se fundamenta en conceptos sólidos. Siendo el hombre un ser social por naturaleza, es muy importante la comunicación, por eso, es fundamental la conceptualización como medio de expresar las ideas y defender, con argumentos, el proyecto o estudio.

Una definición sencilla establece que la argumentación es el "mecanismo que relaciona los datos concretos con las abstracciones y generalizaciones" (Rodriguez, L. 2000). Sin embargo, una definición que nos muestra su complejidad es la siguiente:

La argumentación es un modo de razonamiento que comprende la demostración y la refutación (...) En el proceso de argumentación, desarrollar convicciones en el interlocutor o el auditorio a menudo significa 


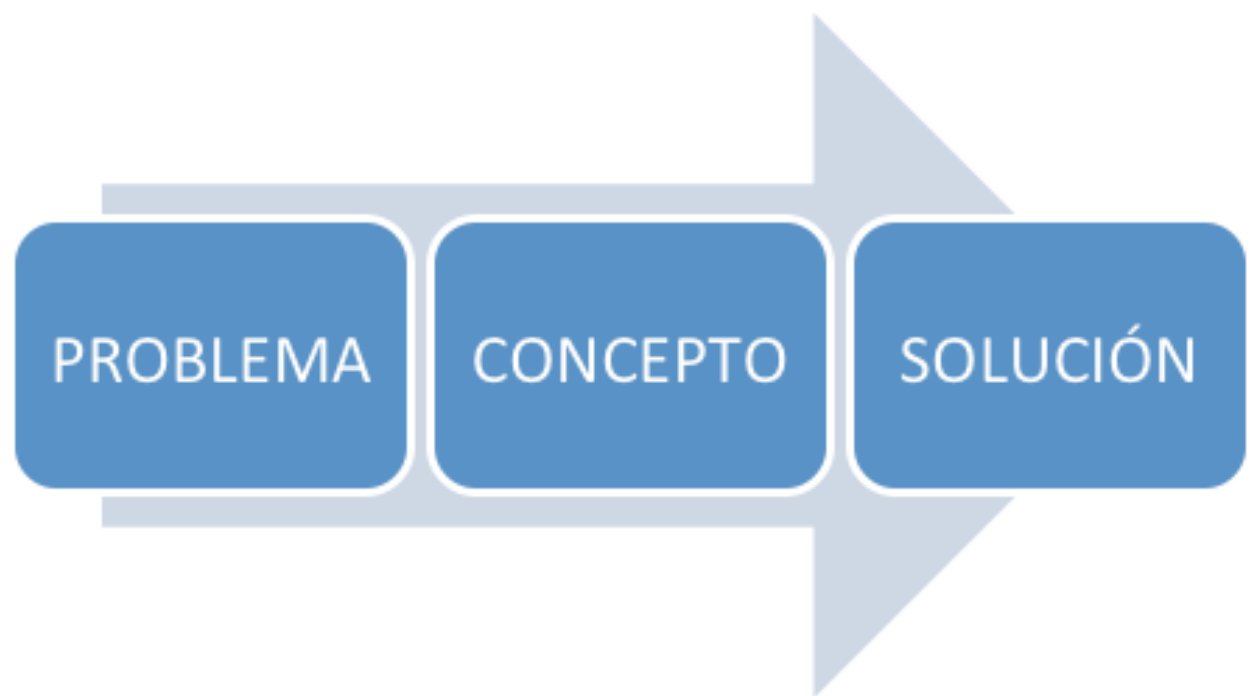

Figura 07 Esquema Conceptos Problémicos.

Fuente: Elaboración propia del autor

hacerlo cambiar de opinión. (...) La comunicación intelectual es imposible sin la argumentación de los enunciados. La argumentación es un instrumento imprescindible para el conocimiento humano de la verdad. (Guétmanova, 1991. Pág. 34).

Del párrafo anterior se desprenden varios elementos importantes: la argumentación necesita de un proceso de razonamiento que se enriquece a través de la demostración y la refutación. Es además un proceso de interacción con el interlocutor. Los enunciados son indispensables para que exista comunicación desde el punto de vistas intelectual. Para el conocimiento de la verdad es indispensable la argumentación.

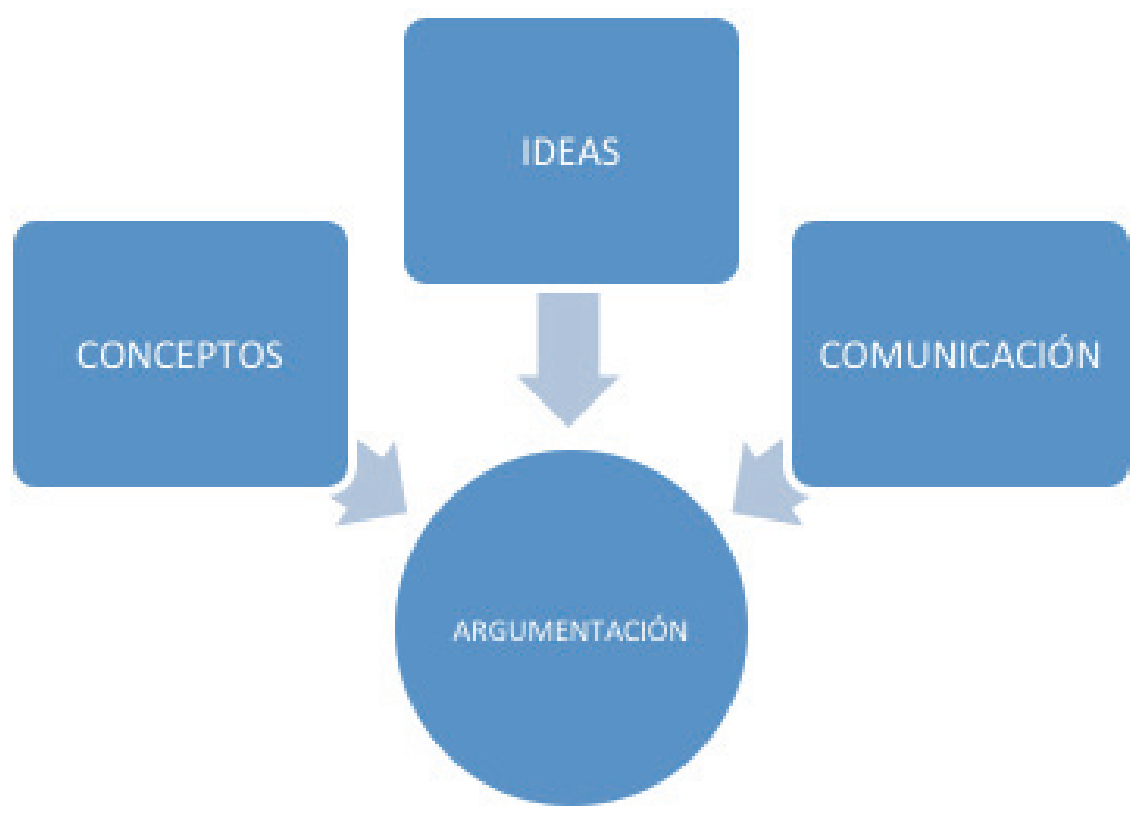

Figura 08 Proceso de argumentación.

Fuente: Elaboración propia del autor 
Entonces, es la argumentación el mecanismo que permite el proceso reflexivo de la conceptualización, que a través de la complementación con las experiencias, permite el desarrollo de las ideas.

Entrelazar los conceptos para formar ideas objetivas, sopesadas en la teoría y complementadas con la práctica, que se puedan expresarse a través de actos comunicativos concretos, da origen a la argumentación, pero todo ello constituye un proceso complejo que involucra el análisis y la reflexión.

El proceso de conceptualización al momento de llegar al diseño arquitectónico se torna más complejo, por las múltiples implicaciones que tiene:

El proyecto de arquitectura constituye una realización intelectual de alta complejidad. El arquitecto al proyectar, coordina aspectos de diversa naturaleza y los sintetiza a través de un sistema de ideas que van expresando relaciones esenciales entre necesidades, intenciones y niveles de satisfacción, tanto materiales como espirituales, de los individuos que harán uso de las obras arquitectónicas y de la sociedad en general. (Cruz, S. sf. Pág. 6).

Sin embargo, la intención sigue siendo la misma, ahora no solo de forma argumentativa sino a través de la creación arquitectónica, comunicarse con la sociedad que valora su obra desde las diversas ópticas.
Si el diseñador articula significados en el proceso de conformación de la arquitectura, es importante que maneje los recursos expresivos propios de su lenguaje a fin de lograr niveles adecuados de comunicación con los usuarios y que estos satisfagan sus expectativas estéticas y artísticas con respecto a la arquitectura. (Cárdenas, 1998. Pág. 151).

El proyecto arquitectónico se debe a la esencia de los sentimientos y sensaciones de sus usuarios, así como al contexto donde se emplaza la obra; su función social, el valor cultural y su representatividad, son aspectos a tener en cuenta al momento de establecer los conceptos para el desarrollo de las ideas que le permitirán la argumentación a través de actos comunicativos de tipo verbal y visual. Con esta serie de elementos, el arquitecto está en condiciones de elegir los conceptos que guiarán el proceso de diseño, no es dotar al diseño de un concepto, es el diseño el resultado del concepto.

Para visibilizar algunos conceptos que se puedan utilizar en la materialización de un proyecto arquitectónico se presenta una relación (Plazola, 1993):

1. Analogía con la naturaleza: En estos diseños predominan las formas orgánicas que están presentes en la naturaleza; a través de un análisis las interpreta y las plasma en el diseño.

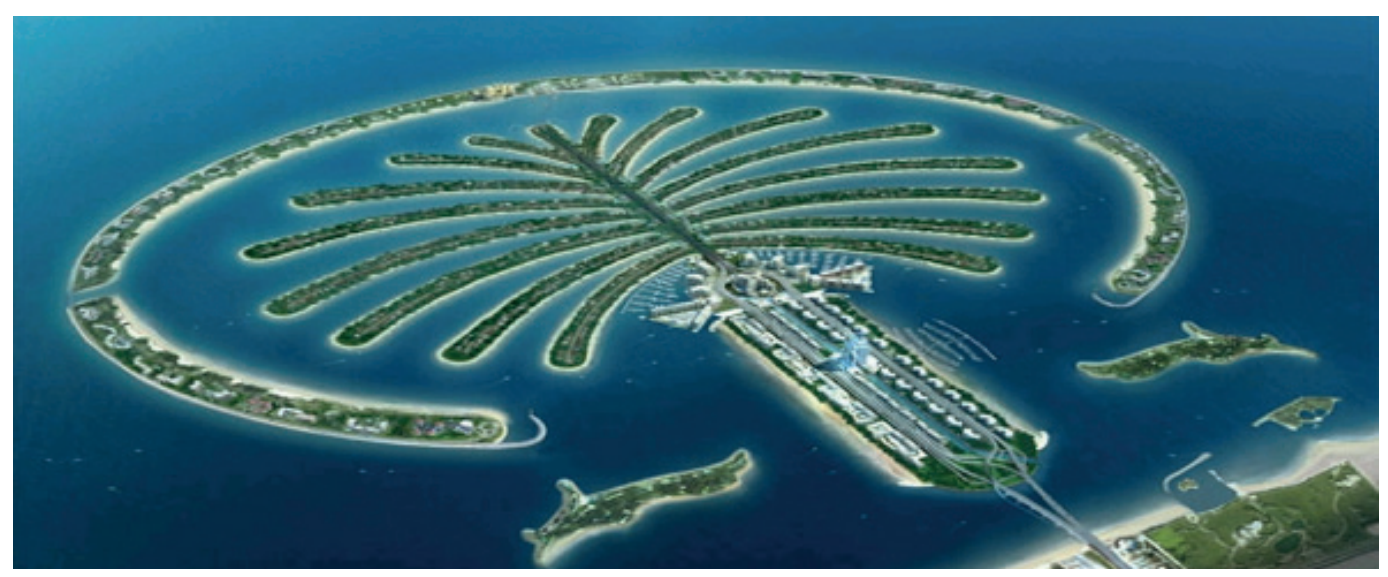

Figura 09 Hotel Dubai.

Fuente: http://www.navarraconfidencial.com/2007/07/14/una-empresa-navarra-iluminara 
2. Analogía con otros proyectos: El punto de partida es un proyecto conocido, el cual es enriquecido con aportes propios logrando un diseño totalmente distinto.

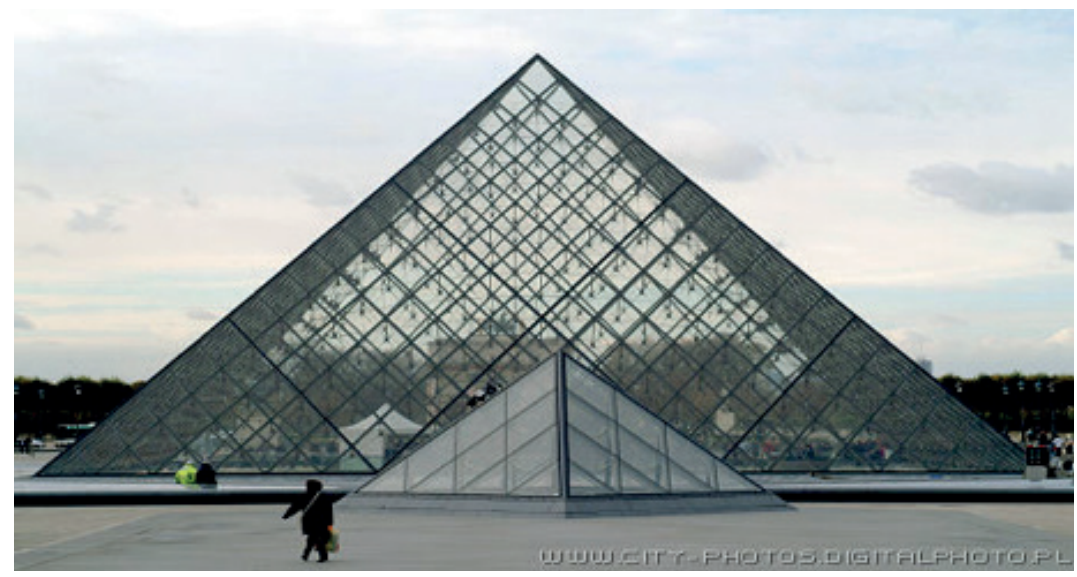

Figura 10 Pirámide De Louvre.

Fuente: http://www.arqhys.com/contenidos/piramide-louvre.html

3. Metáforas formales: Es la relación que se establece entre el proyecto y objetos conocidos sin llegar a copiarlos idénticamente, aunque en ocasiones la similitud sea muy estrecha.

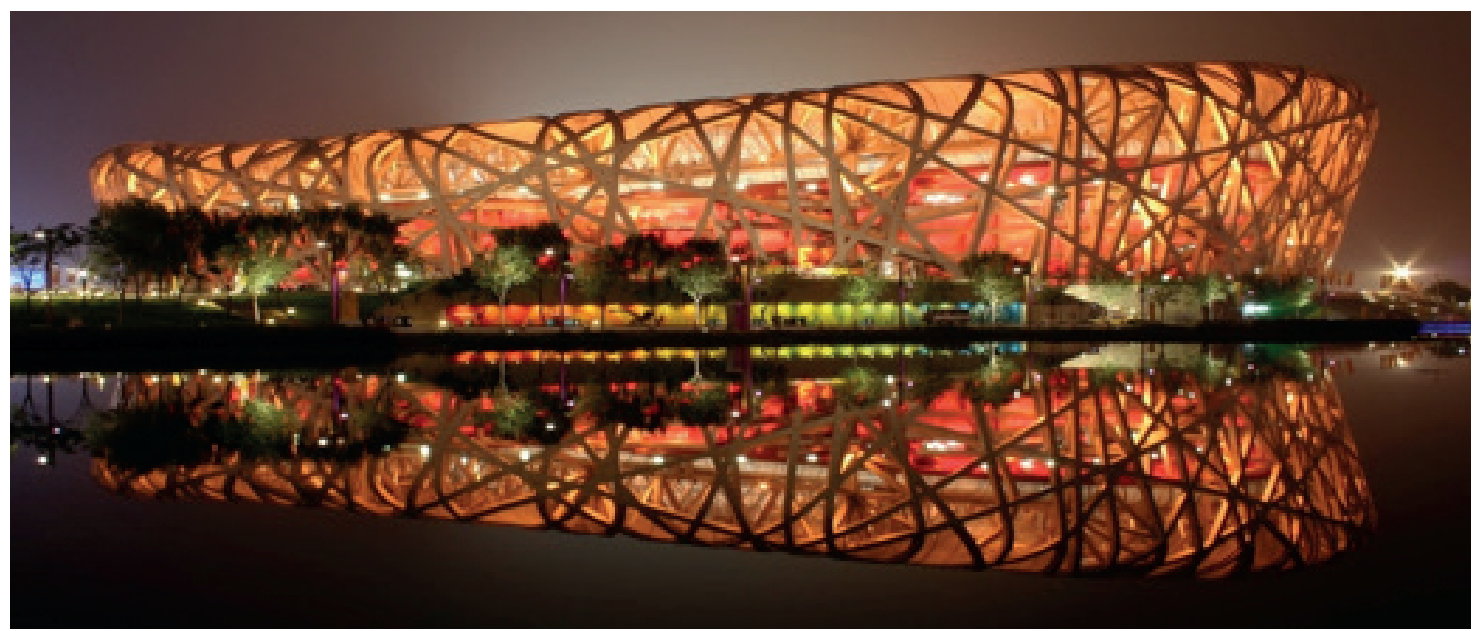

Figura 11 Estadio Nacional de Pekín, El Nido de Pájaro.

Fuente: http://search.babylon.com/imageres.php?iu=http://upload. wikimedia.org/wikipedia/commons/a/a4 Beijing_National_Stadium 
PROCESOS URBANOS - Revista de Divulgación Científica Vol. 1 Enero - Diciembre 2014 (35 - 46)

4. Metáfora de alguna idea: En el proyecto se expresan sentimientos o ideas intangibles, como por ejemplo, amor, tranquilidad, soledad, silencio.

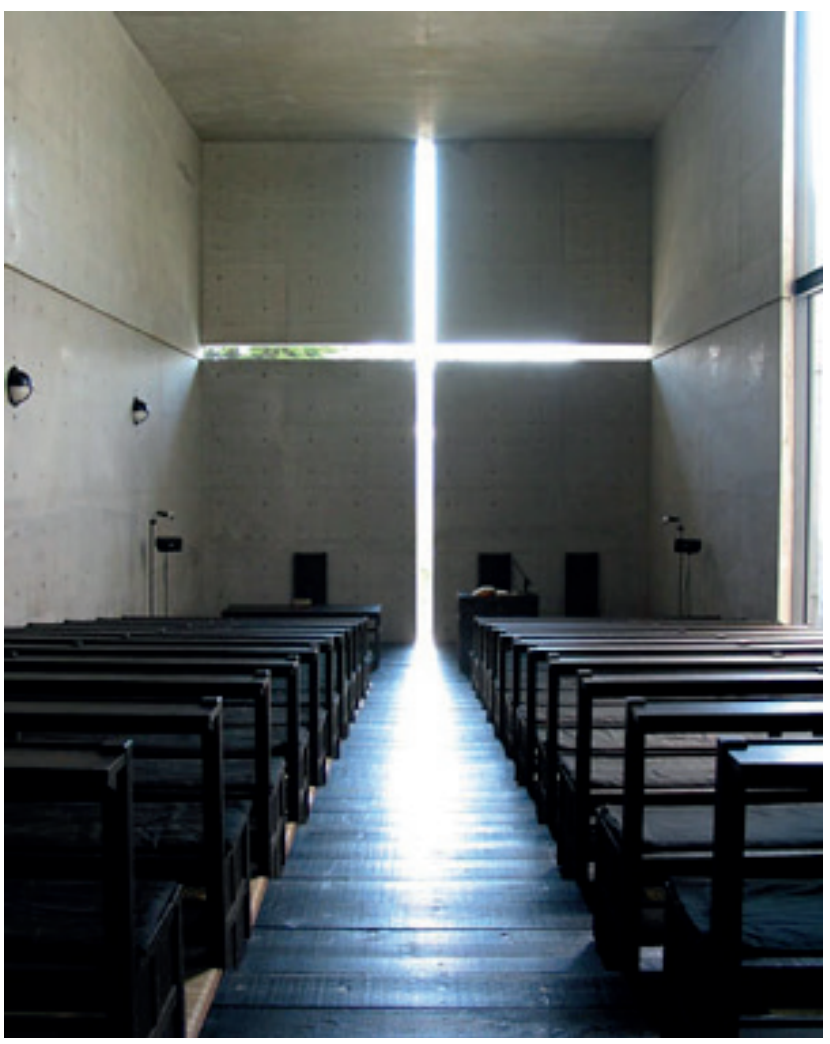

Figura 12 Iglesia de la Luz.

Fuente: http://search.babylon.com/imageres.php?iu=http://3.bp.blogspot.com/

5. Explotación formal: Estos proyectos se caracterizan por un fuerte diseño formal, con formas repetidas y en ocasiones con sensación a rigidez.

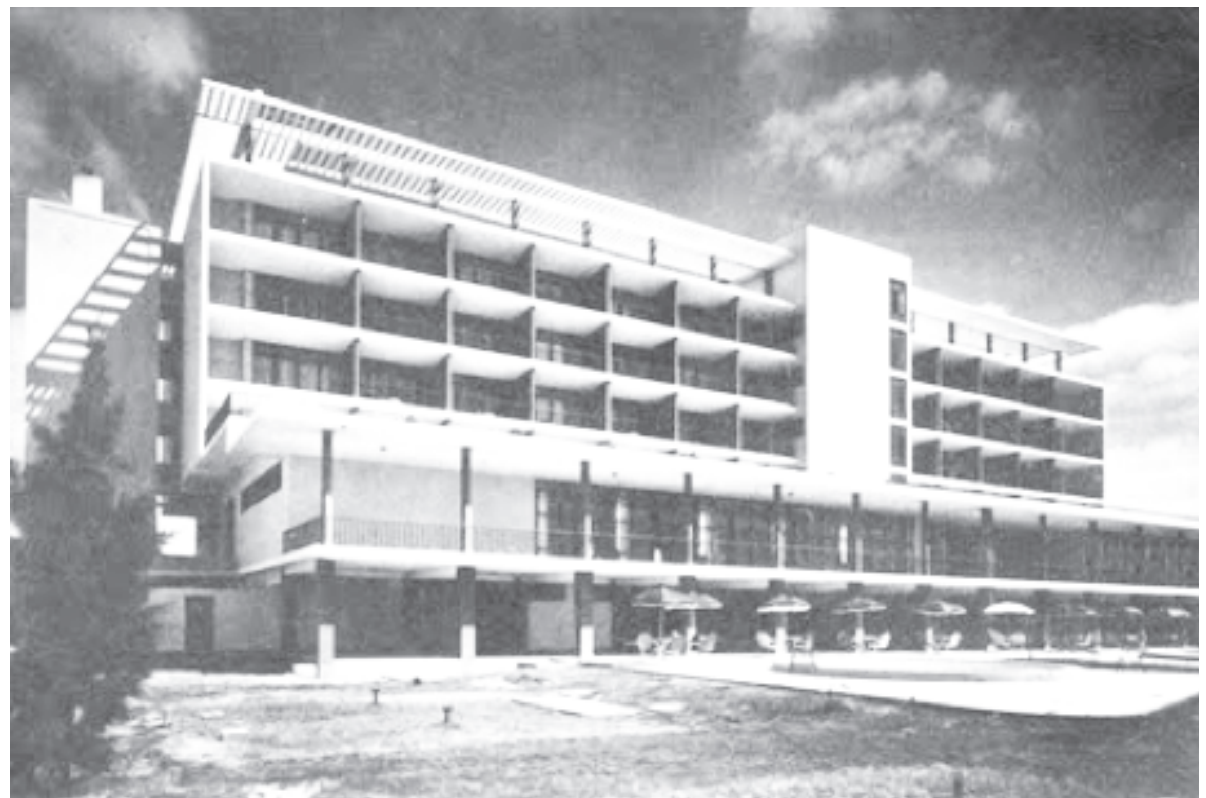

Figura 13 Juan Benites Dubean Hotel de Turistas Fuente: at: http://formamoderna.blogspot.com/ 
6. Inspiraciones vernáculas: Proyectos realizados teniendo como base la arquitectura autóctona tradicional.

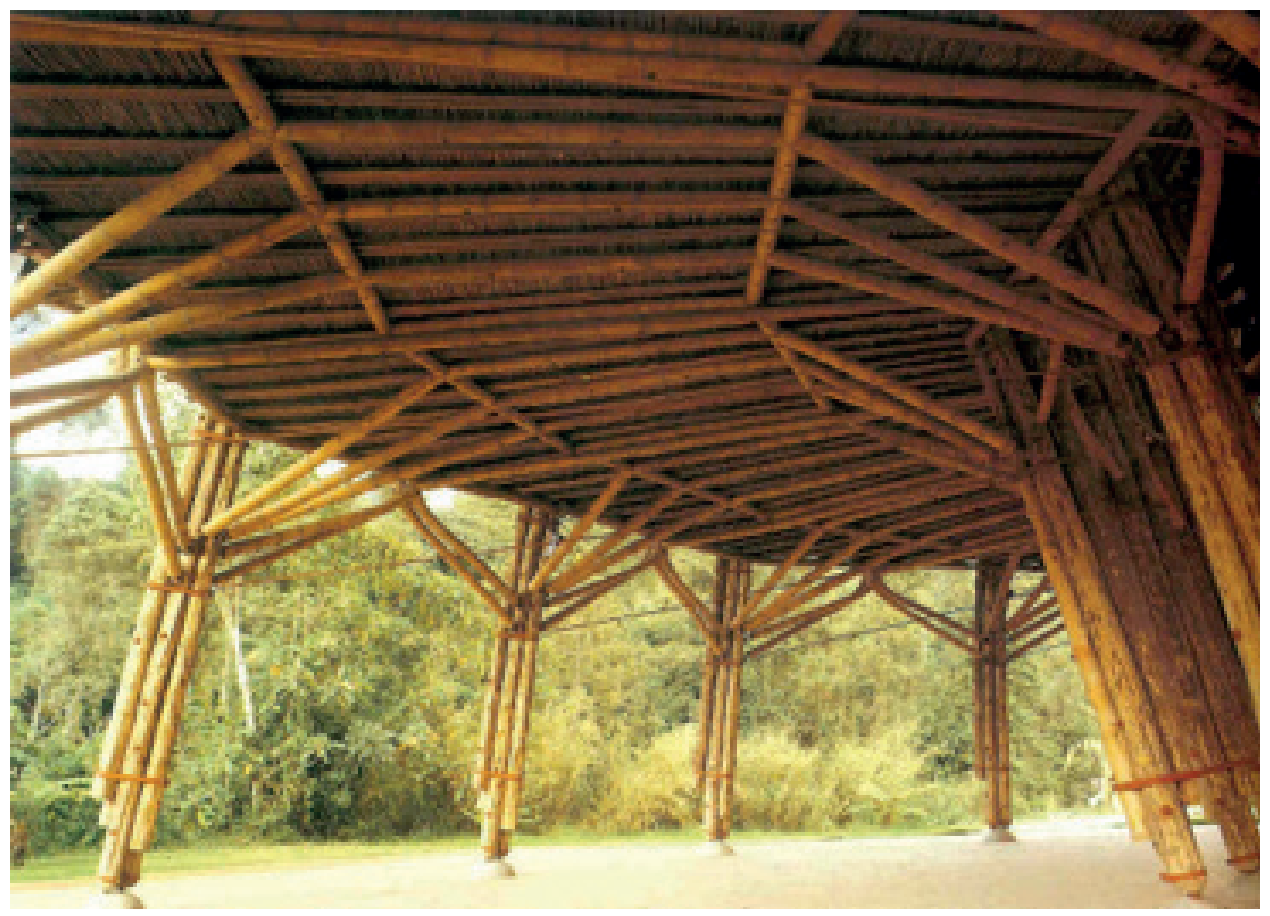

Figura 14 Arquitectura en Guadua. Simón Vélez Fuente: at: http://www.arqsustentable.net/zeri.htm

\section{CONCLUSIONES}

La importancia del concepto nace en el proceso mental reflexivo que permite definir objetos o fenómenos de la sociedad, con los cuales se llega a una instancia superior del pensamiento: las ideas, las cuales permiten explicar las relaciones teóricas, prácticas, metodológicas o simbólicas existentes.

Para entender la significancia del concepto se debe valorar la experiencia como fuente originaria, pero a la vez, se necesita el complemento de los procesos mentales que permiten la generalización.

La importancia de la argumentación surge de la necesidad de comunicación y defensa de las ideas a través del mecanismo de relacionar datos concretos con las abstracciones y generalizaciones. A la verdad sólo se llega por medio de la argumentación.
El diseño arquitectónico es el resultado del concepto y no el proceso inverso, al diseño intentar darle un concepto.

\section{REFERENCIAS}

Cárdenas, E. (1998). Problemas de Teoría de la Arquitectura. México: Universidad de Guanajato.

Cruz, B. S. (s.f.). La conceptualización del proyecto de arquitectura, un ejercicio. universidadypatrimonio.net. Recuperado el 5 de Febrero de 2013, de universidadypatrimonio.net/doc/ doc/1_1_54.pdf

DRAE, D. d. (2013). Real Academia Española, Edición 22. Recuperado el 7 de Abril de 2013, de http://www.rae. es/rae.html 
PROCESOS URBANOS - Revista de Divulgación Científica Vol. 1 Enero - Diciembre 2014 (35 - 46)

Guattari, F. (1999). http://www. hackitectura.net. Recuperado el 23 de Julio de 2013, de http://www. hackitectura.net/osfavelados/2009_ proyectos_eventos/200911_guattari_ lugo/200911_presentacion_lugo_ guattari.pdf

Guétmanova, A. e. (1991). Lógica: en forma simple lo complejo. Moscú: Editorial Progreso.

Martinez, P. (2009). La arquitectura como problema. Sincelejo, Colombia: Ediciones CECAR.
Miranda, M. E. (18 de Diciembre de 2011). El concepto en el diseño arquitectónico (Parte I), Apuntes de arquitectura, Revista digital, $N^{\circ} 34$. Recuperado el 7 de Enero de 2013, de http://apuntesdearquitecturadigital. blogspot.com/2011/12/revistadigital-apuntes-de-arquitectura.html

Plazola, C. A. (1993). Arquitectura habitacional (Vol. (Vol. I)). México: Plazola Editores.

Rodríguez, P. L. (2000). Curso de Español. Universidad para todos. Cuba: Editado por juventud revelde. 